\title{
Effect of L-isoleucine and L-phenylalanine Addition on Aroma Compound Formation During Longan Juice Fermentation by a Co-culture of Saccharomyces cerevisiae and Williopsis saturnus
}

\author{
T. T. T. Trinh ${ }^{1}$, W.Y. Woon ${ }^{1}$, B. Yu², P. Curran², S.-Q. Liu ${ }^{1 *}$ \\ (1) Food Science and Technology Programme, Department of Chemistry, National University of Singapore, 4 Science Drive 4, \\ Singapore 117543 \\ (2) Firmenich Asia Pte Ltd, Tuas, Singapore
}

Submitted for publication: February 2010

Accepted for publication: May 2010

Key words: 2-phenylethyl acetate, active amyl acetate, longan wine, mixed culture, non-Saccharomyces yeast, fermentation, flavour

\begin{abstract}
Some amino acids are known to be precursors of aroma-active compounds produced by yeast metabolism. This study examined the effect of the addition of L-isoleucine and L-phenylalanine on the volatile profiles of longan wine fermented by a co-culture of Saccharomyces cerevisiae var. bayanus EC-1118 and Williopsis saturnus var. saturnus CBS254 inoculated at a ratio of 1:1 $000 \mathrm{cfu} / \mathrm{mL}$ with the aim of enhancing longan wine aroma. Significantly higher concentrations of active amyl alcohol (2-methyl-1-butanol), 2-phenylethyl alcohol and their corresponding acetate esters were found in the longan wine with added L-isoleucine and L-phenylalanine respectively, compared with the control (without added amino acids). The biosynthesis of other volatile compounds was either similar or minimally different, although the formation of some acetate esters, such as isoamyl acetate, hexyl acetate and ethyl acetate, was decreased. These results suggest that the added amino acids play an important role in enhancing the production of targeted aroma compounds in longan wine fermented with mixed yeasts, and that the addition of selected amino acids can be a valuable tool to modulate the formation of aroma compounds in longan wine.
\end{abstract}

\section{INTRODUCTION}

There are many factors that may have an important impact on the formation of aroma compounds in grape and fruit wine, and thus on its quality, including yeast type, the population of individual yeasts in the fruit juice, the characteristic composition of the fruit, juice treatment methods and fermentation conditions (HenickKling et al., 1998; Herrero et al., 2006; Miller et al., 2007; Molina et al., 2007). Meanwhile, fruit wine production practices are largely adopted from the grape wine industry, and new approaches that are suitable for fruit wine need to be developed.

Wine production on an industrial scale often employs sulphur dioxide to kill indigenous bacteria and wild yeasts in the fermentation media before yeast inoculation. These methods, however, compromise wine flavour owing to the formation of undesirable volatile sulphur compounds, aldehydes and ketones, or the loss of natural aroma compounds from the original juice (Henick-Kling et al., 1998). Furthermore, fermentation conditions such as $\mathrm{pH}$, temperature, aeration or nutritional supplementation could also affect the ability of yeasts to produce aroma compounds (Herrero et al., 2006; Molina et al., 2007). The synergistic effects of these factors will result in alcoholic fruit beverages possessing different flavour profiles.

Co-inoculation of non-Saccharomyces and $S$. cerevisiae yeasts into must has recently been suggested as a tool to increase aroma intensity, complexity and fermentation reliability not only for grape wine, but also other fruit wines such as pineapple wine (Panjai et al., 2009). The rationale for this suggestion is that the presence of the non-Saccharomyces yeasts contributes to the increased production of some favourable aroma compounds, such as isoamyl acetate (banana-like aroma), 2-phenylethyl acetate (rose petal-like aroma) and other acetate esters recognised as important flavour compounds in wine and other alcoholic beverages, while $S$. cerevisiae yeasts help improve the levels of alcohol and avoid sluggish fermentation (Rapp \& Mandery, 1986; Rojas et al., 2003).

Non-Saccharomyces yeasts generally develop during the early stages of spontaneous fermentation and their population declines when Saccharomyces yeasts become dominant in the later stages of fermentation (Heras-Vazquez et al., 2003; Maro et al., 2007). As a result, a mixed starter culture of defined yeast ratio should be introduced to control the fermentation process, in which nonSaccharomyces yeasts should not die off early when co-existing with $S$. cerevisiae during fermentation, thus resulting in wine with a wide variety of flavour components. In this context, different yeast ratios have been examined in several studies. For instance, as the proportion of initial Hanseniaspora osmophila inoculum was increased from 0 to $90 \%$ versus $S$. cerevisiae, the production of 2-phenylethyl acetate was enhanced (Viana et al., 2009). On the other hand, wines inoculated with equal ratios of Saccharomyces and non-Saccharomyces yeasts have similar compositions of ethanol, volatile acidity, ethyl acetate and ethyl lactate, but decreased levels of some alcohols when compared with single $S$. cerevisiae-fermented wines (Jolly et al., 2003; Kim et al., 2008). Meanwhile, Rojas et al. (2003) found an increase in acetate ester, but no effect on ethyl ester contents, when mixed cultures of $H$. guilliermondii and Pichia anomala were simultaneously inoculated with $S$. cerevisiae in a ratio of 10:1 ratio respectively. 
Many amino acids are flavour precursors, such as leucine, isoleucine, phenylalanine, valine, serine, threonine and aspartic acid, and most of these are naturally present in fruit juices. However, some kinds of tropical fruits are deficient in either amino acid composition or quantity (Hall et al., 1980). The metabolism of these amino acids by yeasts gives rise to a range of volatile and non-volatile flavour compounds during fermentation. Branchedchain amino acids (leucine, isoleucine and valine) are important in forming not only fusel oils (branched-chain alcohols or higher alcohols), but also branched-chain aldehydes or acids, and most importantly, branched-chain esters. In particular, L-leucine is known to be the precursor of isoamyl alcohol (3-methyl-1-butanol) (Dickinson et al., 1997), with L-valine being the precursor of isobutyl alcohol (2-methyl-1-propanol) (Dickinson et al., 1998) and L-isoleucine being the precursor of active amyl alcohol (2-methyl-1-butanol) (Dickinson et al., 2000). 2-Phenylethanol and 2-phenylethyl acetate, which play a positive role in wine aroma and impart fruity and flowery flavours (Rapp \& Mandery, 1986), are derived from phenylalanine (Etschmann et al., 2002; Dickinson et al., 2003). Other potential flavour compounds that can be derived from this amino acid include benzaldehyde, benzyl acetaldehyde (phenylacetaldehyde), phenylacetic acid and phenyl propanoate. The addition of amino acids into fermentation media to study the production of volatile compounds has been examined in previous studies (Hernandez-Orte et al., 2005; Miller et al., 2007; Garde-Cerdan \& Ancin-Azpilicueta, 2008). Nevertheless, none of them have focused on the effect of individual amino acids on the formation of wine aroma compounds, especially by mixed yeast cultures.

In our study, longan fruit was chosen to make fruit wine because of its rich amount of sugars and significant level of polyphenols, and because it is a good source of ascorbic acid, potassium, copper and other minerals (Rangkadilok et al., 2005; Wall, 2006). Longan wine can thus be considered to be a prospective fruit wine for niche markets. Particularly, longan fruit flesh was found to contain total sugars at $67.35 \mathrm{~g} / 100 \mathrm{~g}$ dry weight, with xylose $(2.94 \%)$, fructose $(2.3 \%)$, glucose $(44.69 \%)$, maltose $(15 \%)$ and sucrose (5.37\%) in its composition (Chang et al., 1998). However, longan is relatively low in those amino acids that act as aroma precursors in wine fermentation, such as leucine, isoleucine and valine $(2.39,1.79$ and $1.54 \mathrm{mg} / 100 \mathrm{~g}$ dry weight of longan fruit flesh respectively) (Chang et al., 1998). As a result, the purpose of the current study was to enhance aroma compound formation in longan wine obtained from the fermentation of longan juice, for which the addition of selected amino acids was carried out, followed by a characterisation of their effects on the volatile flavour compound profile. A high inoculum ratio of a mixed culture of Williopsis saturnus var. saturnus CBS254 and Saccharomyces cerevisiae var. bayanus EC-1118 (1 000:1 cfu/mL) was used due to the latter yeast being the faster fermenter, while the former is able to produce high quantities of esters such as isoamyl acetate (Iwase et al., 1995; Yilmaztekin et al., 2008). The intention was to obtain a contribution from both yeasts.

\section{MATERIALS AND METHODS}

\section{Yeast strains and culturing}

Two yeast strains, Williopsis saturnus var. saturnus CBS254 (CBS Culture Collections, the Netherlands) and Saccharomyces cerevisiae var. bayanus EC-1118 (Lallemand Australia Pty Ltd, Australia), were used. They were maintained in nutrient broth $(0.25 \% \mathrm{w} / \mathrm{v}$ yeast extract, $0.25 \% \mathrm{w} / \mathrm{v}$ peptone, $0.25 \% \mathrm{w} / \mathrm{v}$ malt extract and $2 \% \mathrm{w} / \mathrm{v}$ glucose, $\mathrm{pH} 5.0$ ) and stored in $1 \mathrm{~mL}$ sterile tubes at $-80^{\circ} \mathrm{C}$ as stock cultures before use. Yeast inocula were obtained by growing in sterile longan juice at $25^{\circ} \mathrm{C}$ for one to two days and being co-inoculated into sterile longan juices at a ratio of EC-1118:CBS254 = 1:1 $000\left(10^{2}\right.$ and $10^{5} \mathrm{cfu} / \mathrm{mL}$ respectively $)$.

\section{Preparation of sterile longan juice}

Fresh longan fruits (Dimocarpus longan Lour.) purchased from the local market were peeled, the seeds were removed, they were crushed and then squeezed through a clean cloth to obtain raw juice. The juice was stored at $-20^{\circ} \mathrm{C}$ before use, which can prolong the shelf life without changing the composition of the juice into a soluble solids concentration and titratable acidity (Jiang, 1999). The longan juice was sterilised by aseptic micro-filtration through a $0.45 \mu \mathrm{m}$ pore size micro-filter (Sartorius, Germany), which can retain the yeast cells and thus remove wild yeasts initially present in the juice (Chandler \& Zydney, 2005). Furthermore, a sterility check was done by plate counting. Before sterilisation, raw longan juice (about $\mathrm{pH}$ 6.9) was adjusted to $\mathrm{pH} 3.6$ with 50\% $(\mathrm{w} / \mathrm{v})$ L-malic acid solution, followed by centrifugation at 21000 rpm and $4^{\circ} \mathrm{C}$ for 15 min (Allegra ${ }^{\circledR}$ 64R High-Speed Refrigerated Benchtop Centrifuge, Beckman Coulter, UK), and pre-filtration of the supernatant through a $0.65 \mu \mathrm{m}$ pore size pre-filter (Sartorius, Göttingen, Germany).

\section{Fermentation and determination of yeast growth}

Fermentations were carried out in $250 \mathrm{~mL}$ conical flasks covered with cotton wool and aluminium foil, containing $200 \mathrm{~mL}$ of sterile longan juice. The operating temperature was maintained at $20^{\circ} \mathrm{C}$ for 21 days under static conditions. Three fermentations (each in replicate) were performed, including a control (without added with amino acid), $0.05 \%$ (w/v) L-isoleucine-added and $0.05 \%$ (w/v) L-phenylalanine-added longan juices. The amino acids used were purchased from Sigma-Aldrich. During the fermentation the yeasts were enumerated by the spread plating method on potato dextrose agar (Oxoid Ltd, Basingstoke, Hampshire, UK). The differentiation of yeasts was based on a combination of incubation temperature and their colony appearances: $25^{\circ} \mathrm{C}$ for the growth of both yeasts and $35^{\circ} \mathrm{C}$ for the growth of $S$. cerevisiae EC-1118, whose colony was creamy, shiny and smooth while that of strain CBS254 appeared dull, wrinkled and rough. All equipment in contact with the juice was autoclaved at $121^{\circ} \mathrm{C}$ for $15 \mathrm{~min}$.

\section{Analytical methods}

Twenty-mL aliquots of samples were taken aseptically after gently swirling the conical flasks. Analytical parameters, including $\mathrm{pH}$, Brix and optical density at $600 \mathrm{~nm}$ wavelength, were then determined. The remaining samples were centrifuged at $5000 \mathrm{rpm}$, $4^{\circ} \mathrm{C}$ for $15 \mathrm{~min}$ in capped tubes to remove yeast cells. The cellfree supernatants were stored at $-20^{\circ} \mathrm{C}$ for the analysis of volatile compounds. All determinations were conducted in duplicate.

\section{Gas chromatography-mass spectrometry (GC-MS) analysis}

Headspace compounds of $5 \mathrm{~mL}$ samples contained in $20 \mathrm{~mL}$ glass vials tightly capped with a PTFE/silicone septum were extracted by the SPME $85 \mu \mathrm{m}$ carboxen/polydimethylsiloxane (Supelco, Missouri, USA) for $30 \mathrm{~min}$ at $60^{\circ} \mathrm{C}$. The fibre was then injected into an HP 6890 gas chromatograph coupled to a flame ionisation 
detector (FID) and an HP 5973 mass spectrometry detector (MSD, Agilent Technology, USA) in splitless mode. An Agilent DB-FFAP capillary column ( $60 \mathrm{~m} \times 0.25 \mathrm{~mm}$ I.D. $\mathrm{x} 0.25 \mu \mathrm{m}$ film thickness $)$ was used for chromatographic separation. The temperature of the $\mathrm{GC}$ oven was programmed as follows: $50^{\circ} \mathrm{C}$ for $4 \mathrm{~min}$, then increased to $230^{\circ} \mathrm{C}$ at a rate of $5^{\circ} \mathrm{C} \mathrm{min}^{-1}$, and maintained at $230^{\circ} \mathrm{C}$ for $20 \mathrm{~min}$. The FID temperature was set at $250^{\circ} \mathrm{C}$, and the MSD was operated in the electron impact mode at $70 \mathrm{eV}$. Identification of the eluted compounds was achieved by matching their mass spectra against the NIST 8.0 MS library (National Institute of Standards and Technology, Gaithersburg, MD, USA) and confirmed with linear retention index (LRI) values. LRI values on the FFAP column were determined using a series of alkanes (C5-C25) run under identical conditions. Several volatiles were selected to be quantified using external standards dissolved in $10 \% \mathrm{v} / \mathrm{v}$ micro-filtered $(0.45 \mu \mathrm{m})$ longan juice diluted with water, except for ethanol dissolved in $100 \% \mathrm{v} / \mathrm{v}$ micro-filtered longan juice. Analyses were carried out in duplicate.

\section{Statistical analysis}

Tukey's HSD test (software R for Windows, version 2.9.1) was used for the analysis of variance of the GC-FID peak areas obtained on day 21 . The results represent the mean values and standard deviations calculated from the data of the two independent fermentations.

\section{RESULTS AND DISCUSSION}

\section{Yeast growth, changes in total soluble solids and $\mathrm{pH}$ during longan juice co-fermentation}

The kinetics of yeast growth, total soluble solids as well as the $\mathrm{pH}$ of longan juice fermented by a mixed culture of $S$. cerevisiae EC1118 and $W$. saturnus CBS254 showed similarity disregarding added isoleucine or phenylalanine (Fig. 1), which may explain the similar ethanol concentrations obtained in the control and amino acid-added fermented longan juice at the end of fermentation (6.94 to $7.33 \% \mathrm{v} / \mathrm{v}$, Table 1). The strain CBS254 reached its stationary phase by day 6 , and then the viable cell count remained essentially unchanged. Strain EC-1118 grew consistently during fermentation, although its cell count was lower than that of strain CBS254 until day 21. Consequently, the sugar level showed a slow decrease due to $W$. saturnus CBS254 being a weak sugar fermenter (approximately 27 to $28 \%$ of the initial sugar was consumed). The $\mathrm{pH}$ values of the co-fermented longan juices increased moderately from 3.61 to 3.85 (Fig. 1).

The high inoculation ratio (1 000 -fold) of $W$. saturnus to $S$. cerevisiae used in this study may explain the lack of inhibition of the non-Saccharomyces yeast $W$. saturnus by the Saccharomyces yeast. This finding is in contrast with reports elsewhere (Egli et al., 1998; Pérez-Nevado et al., 2006; Viana et al., 2009). In these studies, non-Saccharomyces yeasts showed an early death in co-cultures with $S$. cerevisiae that are known to dominate and complete the fermentation when they are present spontaneously or simultaneously inoculated in the fermentation media at different ratios, such as 1:1, 1:10 and 1:100 (Saccharomyces : nonSaccharomyces) (Jolly et al., 2003; Rojas et al., 2003; Kim et al., 2008; Viana et al., 2009). This phenomenon has been attributed to many factors, such as fermentation metabolites (e.g. ethanol, short- to medium-chain fatty acids, etc.), yeast killer activity, nutritional limitation and a cell-cell-contact-mediated mechanism
(Hansen et al., 2001; Nissen et al., 2003; Pina et al., 2004; PérezNevado et al., 2006).

\section{Kinetic changes in volatile compounds during longan juice co- fermentation}

Figs. 2 to 6 show the production of a diversity of volatile compounds by the mixed culture of $S$. cerevisiae EC-1118 and $W$. saturnus CBS254 in longan juice, as well as the complex trends induced by these yeasts during co-fermentation.

As shown in Fig. 2, most of the acetate esters reached their maximum by day 3 and then declined. The supplementation of L-isoleucine significantly increased the production of active amyl acetate (2-methyl-1-butyl acetate) (Fig. 2, Table 1). However, the production of butyl acetate and hexyl acetate was reduced and the formation of 2-phenylethyl acetate was unaffected, compared with the control (Fig. 2, Table 1). Likewise, relative to the control, the addition of L-phenylalanine markedly enhanced the production of 2-phenylethyl acetate while decreasing the synthesis of other acetate esters, including methyl acetate, butyl acetate, hexyl acetate and isoamyl acetate, except for isobutyl acetate and active amyl acetate, whose changes were similar (Fig. 2, Table 1). The observed reduction in the formation of methyl, butyl, isoamyl, and hexyl acetates in the presence of added amino acids might be attributable to acetyl-CoAs (known as one of the precursors of acetate ester production catalysed by alcohol acetyltransferases; Yoshioka \& Hashimoto, 1981) being diverted to the biosynthesis of amyl acetates and 2-phenylethyl acetate, which was related to the metabolism of isoleucine and phenylalanine respectively.

The effect of isoleucine and phenylalanine on the kinetics of ethyl esters is presented in Fig. 3. Indigenous ethyl-3-hydroxy butanoate, ethyl butanoate and ethyl-2-butenoate were degraded by the mixed yeasts in the fermented longan juice irrespective of the presence or absence of amino acids. Ethyl acetate initially increased and then decreased, but its synthesis was lower in the fermented juice with added isoleucine or phenylalanine, possibly due to the reason mentioned above for acetate esters. On the contrary, ethyl hexanoate and ethyl octanoate were increasingly produced until day 21 . These two esters showed reduced production in the presence of added phenylalanine when compared with the control.

Among the alcohols that were consistently produced by the co-culture of yeasts EC-1118 and CBS254, active amyl alcohol and 2-phenylethyl alcohol were produced in remarkably higher amounts when isoleucine and phenylalanine were added respectively (Fig. 4, Table 1). Conversely, the addition of the two amino acids resulted in, to a small extent, lessened production of other alcohols, including ethanol, isobutyl alcohol and isoamyl alcohol, except for linalool, which decreased continuously. The enhanced synthesis of active amyl alcohol and 2-phenylethyl alcohol were in agreement with previous studies in which isoleucine and phenylalanine were catabolised by yeasts to form the corresponding alcohols (Dickinson et al., 2000, 2003). These two alcohols serve as precursors for the biosynthesis of active amyl acetate and 2-phenylethyl acetate catalysed by alcohol acetyltransferase (Yoshioka \& Hashimoto, 1981), which explains the greater amounts of these esters in longan juice co-fermentation with the added isoleucine and phenylalanine respectively.

The kinetic changes in the fatty acids, namely acetic, hexanoic, octanoic and decanoic acid, are shown in Fig. 5. Most of the acids 
TABLE 1

Volatile compounds produced by a co-culture of $S$. cerevisiae EC-1118 : W. saturnus CBS254 (ratio of 1:1 $000 \mathrm{cfu} / \mathrm{mL}$ ) in longan wine with added isoleucine and phenylalanine on day 21.

\begin{tabular}{|c|c|c|c|c|c|}
\hline \multirow{2}{*}{ No } & \multirow{2}{*}{ Volatile compound } & \multirow{2}{*}{ LRI } & \multicolumn{3}{|c|}{ Mean \pm standard deviation $\left(10^{6}\right.$ GC-FID peak area $)$} \\
\hline & & & Control LW $\mathbf{W}^{(1)}$ & IsoLeu LW(2) & PheAla $L^{(3)}$ \\
\hline & Acetate esters & & & & \\
\hline 1 & Methyl acetate & 865 & $0.42 \pm 0.04^{\mathrm{a}}$ & $0.48 \pm 0.07^{\mathrm{a}}$ & $0.45 \pm 0.06^{\mathrm{a}}$ \\
\hline 2 & Isobutyl acetate & 1028 & $3.44 \pm 0.38^{\mathrm{a}}$ & $3.69 \pm 0.43^{\mathrm{a}}$ & $4.14 \pm 0.61^{\mathrm{a}}$ \\
\hline 3 & Butyl acetate & 1074 & $0.15 \pm 0.03^{\mathrm{a}}$ & $0.10 \pm 0.01^{\mathrm{a}}$ & $0.11 \pm 0.02^{\mathrm{a}}$ \\
\hline 4 & Active amyl acetate* & 1113 & $\begin{array}{c}0.18 \pm 0.04^{\mathrm{a}} \\
(6.4 \pm 0.4)\end{array}$ & $\begin{array}{l}0.35 \pm 0.01^{\mathrm{b}} \\
(10.1 \pm 3.8)\end{array}$ & $\begin{array}{c}0.11 \pm 0.02^{\mathrm{c}} \\
(4.9 \pm 1.9)\end{array}$ \\
\hline 5 & Isoamyl acetate* & 1123 & $\begin{array}{l}17.78 \pm 2.62^{\mathrm{b}} \\
(2302 \pm 101)\end{array}$ & $\begin{array}{l}11.16 \pm 1.26^{\mathrm{a}} \\
(1396 \pm 135)\end{array}$ & $\begin{array}{l}9.80 \pm 1.43^{\mathrm{a}} \\
(1327 \pm 89)\end{array}$ \\
\hline 6 & Hexyl acetate & 1296 & $0.53 \pm 0.05^{\mathrm{a}}$ & $0.42 \pm 0.06^{\mathrm{b}}$ & $0.31 \pm 0.05^{\mathrm{c}}$ \\
\hline \multirow[t]{2}{*}{7} & 2-Phenylethyl acetate* & 1873 & $\begin{array}{c}178.33 \pm 22.18^{\mathrm{a}} \\
(4079 \pm 411)\end{array}$ & $\begin{array}{c}166.63 \pm 7.71^{\mathrm{a}} \\
(4024 \pm 366)\end{array}$ & $\begin{array}{c}378.19 \pm 49.93^{b} \\
(11801 \pm 326)\end{array}$ \\
\hline & Ethyl esters & & & & \\
\hline 8 & Ethyl acetate & 922 & $181.42 \pm 44.43^{\mathrm{a}}$ & $119.08 \pm 1.86^{\mathrm{b}}$ & $153.31 \pm 16.25^{\mathrm{ab}}$ \\
\hline 9 & Ethyl butanoate & 1046 & $4.83 \pm 0.84^{\mathrm{a}}$ & $4.82 \pm 0.37^{\mathrm{a}}$ & $4.39 \pm 0.62^{\mathrm{a}}$ \\
\hline 10 & Ethyl 2-butenoate & 1176 & $0.22 \pm 0.03^{b}$ & $0.13 \pm 0.02^{\mathrm{a}}$ & $0.09 \pm 0.02^{\mathrm{a}}$ \\
\hline 11 & Ethyl-3-hydroxy butanoate & 1557 & $0.17 \pm 0.02^{\mathrm{a}}$ & $0.13 \pm 0.01^{\mathrm{b}}$ & $0.09 \pm 0.01^{\mathrm{c}}$ \\
\hline 12 & Ethyl hexanoate & 1246 & $2.53 \pm 0.56^{\mathrm{a}}$ & $2.59 \pm 0.55^{\mathrm{a}}$ & $1.54 \pm 0.10^{\mathrm{b}}$ \\
\hline \multirow[t]{2}{*}{13} & Ethyl octanoate* & 1463 & $\begin{array}{l}12.06 \pm 1.80^{\mathrm{a}} \\
(33 \pm 2)\end{array}$ & $\begin{array}{l}12.29 \pm 2.59^{\mathrm{a}} \\
(40 \pm 6)\end{array}$ & $\begin{array}{l}10.13 \pm 0.51^{\mathrm{a}} \\
\quad(29 \pm 3)\end{array}$ \\
\hline & Alcohols & & & & \\
\hline 14 & Ethanol* & 965 & $\begin{array}{c}3266.54 \pm 152.10^{\mathrm{a}} \\
(7.261 \pm 0.490)\end{array}$ & $\begin{aligned} 3507.78 & \pm 213.04^{\mathrm{a}} \\
(7.333 & \pm 0.273)\end{aligned}$ & $\begin{array}{c}2664.88 \pm 380.64^{b} \\
(6.937 \pm 0.587)\end{array}$ \\
\hline 15 & Isobutyl alcohol & 1093 & $16.04 \pm 0.57^{\mathrm{a}}$ & $14.11 \pm 0.34^{\mathrm{a}}$ & $11.31 \pm 1.94^{\mathrm{b}}$ \\
\hline 16 & Active amyl alcohol* & 1221 & $\begin{array}{c}5.88 \pm 0.61^{\mathrm{a}} \\
(1549 \pm 137)\end{array}$ & $\begin{array}{l}17.84 \pm 0.90^{\mathrm{b}} \\
(4011 \pm 130)\end{array}$ & $\begin{array}{c}4.00 \pm 0.47^{c} \\
(1077 \pm 113)\end{array}$ \\
\hline 17 & Isoamyl alcohol* & 1230 & $\begin{array}{c}21.36 \pm 1.91^{\mathrm{a}} \\
(23623 \pm 2800)\end{array}$ & $\begin{array}{c}20.36 \pm 1.52^{\mathrm{a}} \\
(23386 \pm 2475)\end{array}$ & $\begin{array}{c}15.48 \pm 0.71^{\mathrm{b}} \\
(22721 \pm 2511)\end{array}$ \\
\hline 18 & Linalool & 1580 & $3.30 \pm 0.14^{\mathrm{a}}$ & $3.49 \pm 0.30^{\mathrm{a}}$ & $4.46 \pm 0.40^{\mathrm{b}}$ \\
\hline \multirow[t]{2}{*}{19} & 2-phenylethyl alcohol* & 1975 & $\begin{array}{c}37.41 \pm 12.49^{\mathrm{a}} \\
(22819 \pm 1263)\end{array}$ & $\begin{array}{c}46.25 \pm 6.47^{\mathrm{a}} \\
(28135 \pm 1240)\end{array}$ & $\begin{array}{c}111.87 \pm 10.91^{\mathrm{b}} \\
(104659 \pm 1442)\end{array}$ \\
\hline & Organic acids & & & & \\
\hline 20 & Acetic acid & 1489 & $29.84 \pm 5.91^{\mathrm{a}}$ & $28.96 \pm 3.87^{\mathrm{a}}$ & $25.68 \pm 4.75^{\mathrm{a}}$ \\
\hline 21 & Hexanoic acid & 1885 & $1.39 \pm 0.11^{\mathrm{a}}$ & $1.25 \pm 0.15^{\mathrm{a}}$ & $1.24 \pm 0.10^{\mathrm{a}}$ \\
\hline 22 & Octanoic acid* & 2110 & $\begin{array}{c}3.98 \pm 0.34^{\mathrm{a}} \\
(441 \pm 25)\end{array}$ & $\begin{array}{c}4.03 \pm 0.31^{\mathrm{a}} \\
(452 \pm 35)\end{array}$ & $\begin{array}{c}3.69 \pm 0.39^{\mathrm{a}} \\
(439 \pm 16)\end{array}$ \\
\hline \multirow[t]{2}{*}{23} & Decanoic acid & 2329 & $1.65 \pm 0.42^{\mathrm{a}}$ & $1.55 \pm 0.22^{\mathrm{a}}$ & $1.88 \pm 0.24^{\mathrm{a}}$ \\
\hline & Aldehydes & & & & \\
\hline 24 & Acetaldehyde & 852 & $20.91 \pm 6.24^{\mathrm{a}}$ & $25.27 \pm 2.98^{\mathrm{a}}$ & $22.10 \pm 1.09^{\mathrm{a}}$ \\
\hline 25 & 2-Butenal & 1057 & $5.24 \pm 0.15^{\mathrm{a}}$ & $5.65 \pm 0.61^{\mathrm{a}}$ & $4.71 \pm 0.61^{\mathrm{a}}$ \\
\hline 26 & Benzaldehyde & 1576 & $0.14 \pm 0.01^{\mathrm{a}}$ & $0.15 \pm 0.01^{\mathrm{a}}$ & $0.10 \pm 0.02^{\mathrm{b}}$ \\
\hline
\end{tabular}

Values with the same superscript letters are not significantly different according to Tukey's HSD at the 95\% confidence level $(\mathrm{n}=4)$.

(1) Longan juice co-fermentation without added amino acids

(2) (3) Longan juice co-fermentations with added L-isoleucine and L-phenylalanine respectively.

* Selected volatile compounds for quantification, values shown in brackets are mean concentration \pm standard deviation with unit in $\mu \mathrm{g} \mathrm{L}^{-1}$, except for ethanol in $\%$ ( $/ \mathrm{v}$ ) 


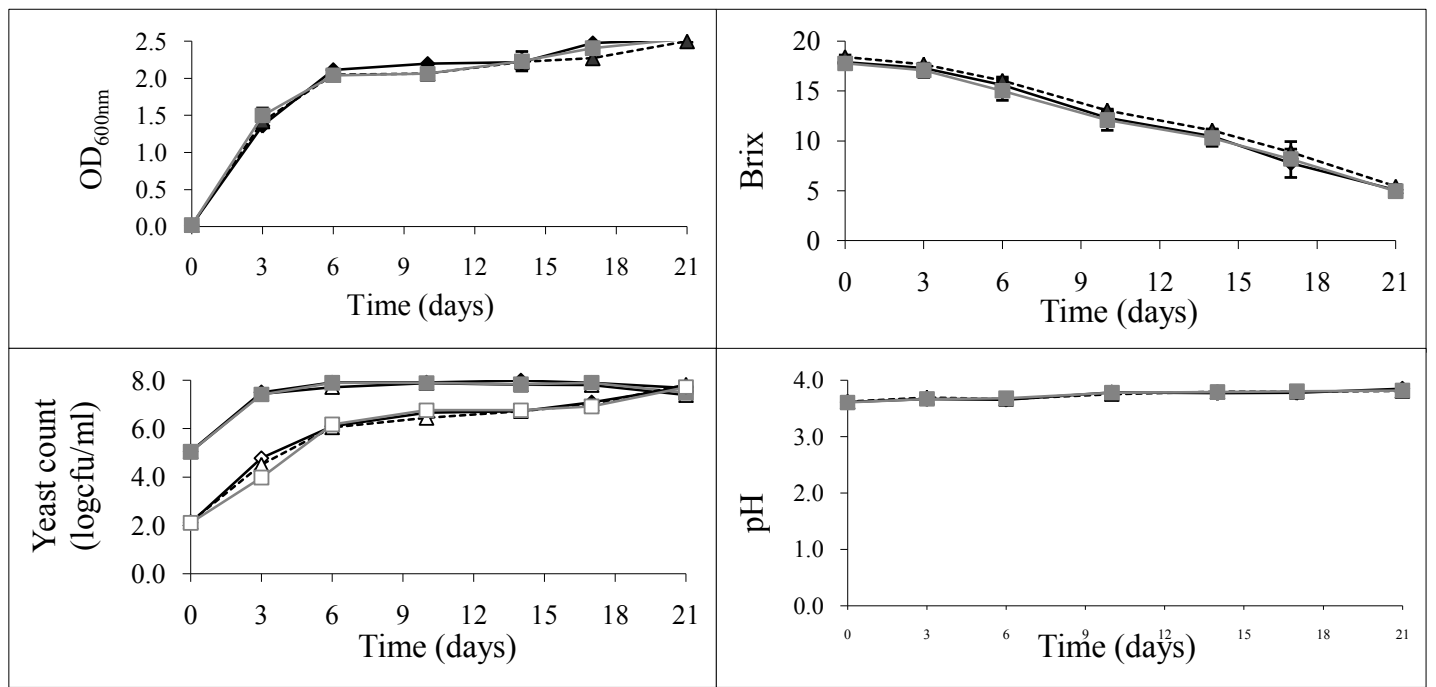

\section{FIGURE 1}

Growth of yeasts (as cell count and $\mathrm{OD}_{600 \mathrm{~mm}}$ ), Brix and $\mathrm{pH}$ changes during longan juice co-fermentation by S. cerevisiae var. bayanus EC-1118 and W. saturnus var. saturnus CBS254 (ratio of 1:1 $000 \mathrm{cfu} / \mathrm{mL}$ respectively) with and without added amino acids. Longan juice without added amino acid (control) ( $\bullet$ ), longan juice with added L-isoleucine ( $(\mathbf{\Delta})$, longan juice with added L-phenylalanine ( $\mathbf{\square})$; EC-1118 (open nodes), CBS254 (filled nodes).

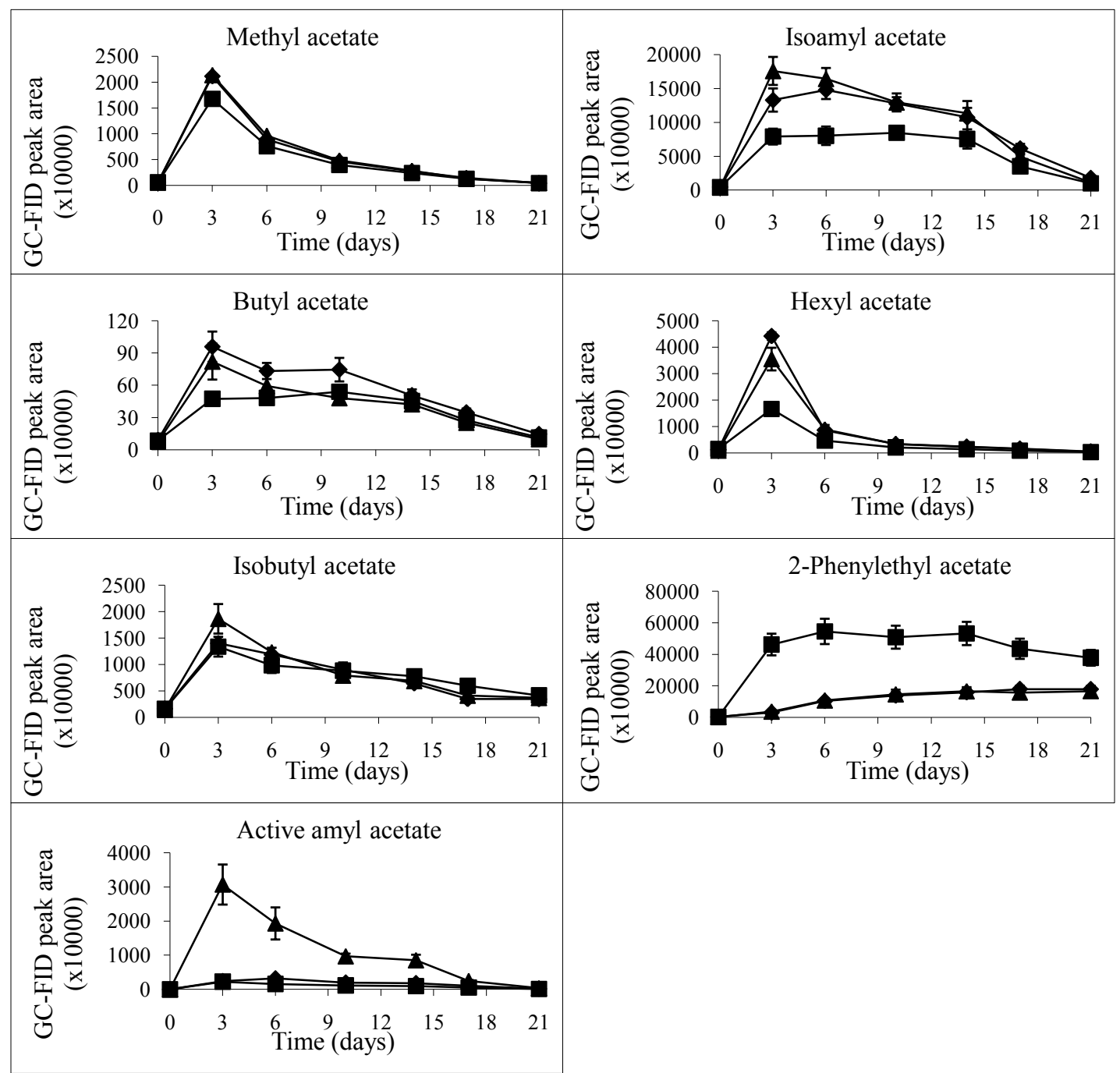

FIGURE 2

Kinetics of acetate esters during longan juice co-fermentation by S. cerevisiae var. bayanus EC-1118 and W. saturnus var. saturnus $\mathrm{CBS} 254$ (ratio of 1:1 000 cfu/mL respectively). Longan juice without added amino acid (control) $(\checkmark)$, longan juice with added L-isoleucine $(\boldsymbol{\Delta})$, longan juice with added L-phenylalanine $(\boldsymbol{\square})$. 
initially increased, and then remained stable before continuing their production, except for acetic acid, which rose incessantly over time. However, the presence of added isoleucine or phenylalanine appeared to have no influence on the formation of these fatty acids (Table 1).

Fig. 6 describes the dynamics of aldehydes, among which acetaldehyde and 2-butenal displayed a slight initial decrease, followed by a consistent increase, but no difference was observed in the presence or absence of isoleucine and phenylalanine (Table 1). Benzaldehyde, which generally reduced, was slightly affected by the addition of phenylalanine.

Volatile compounds in longan wine at the end of co-fermentation with and without added isoleucine and phenylalanine

Most of the volatile compounds identified in longan wine (Table 1) are known to impart sensory properties in grape and other fruit wines, such as apple wine and orange wine (Rapp \& Mandery, 1986; Herrero et al., 2006; Selli et al., 2008). The acetate esters of higher alcohols, such as ethyl acetate, hexyl acetate, isoamyl acetate and 2-phenylethyl acetate, generally give rise to the strong fruity and flowery aroma, while the ethyl esters of fatty acids present pleasant odours of wax or honey - depending on the carbon-chain length of the fatty acids, with the short-chain ones being more desirable (Ribéreau-Gayon et al., 2000). Higher alcohols, which are considered to contribute positively to the complexity of wine flavour below $300 \mathrm{mg} / \mathrm{L}$, exert a negative effect on wine flavour at over $400 \mathrm{mg} / \mathrm{L}$ (Rapp \& Mandery, 1986). Similarly, acetic acid and aldehydes can induce unpleasant odours at levels near their flavour thresholds (Lambrechts \& Pretorius, 2000). The production of these flavour compounds during longan juice fermentation was associated with yeast metabolism; however, ethyl 2-butenoate, ethyl 3-hydroxy butanoate, linalool and benzaldehyde are naturally present in longan juice and were not produced by yeasts in longan wine (see above). and thus were affected less by the addition of isoleucine and phenylalanine (Chang et al., 1998, Zhang et al., 2008a). Biochemically, yeasts can catabolise amino acids by different metabolic pathways. Leucine, valine and phenylalanine are first deaminated to yield $\alpha$-keto acids, followed by decarboxylation to form aldehydes, which are subsequently reduced to form the isoamyl, isobutyl and 2-phenylethyl alcohols respectively (Dickinson et al., 1997, 1998). However, isoleucine can undergo four possible routes, all of which include the initial deamination of isoleucine to $\alpha$-keto- $\beta$ methylvalerate, followed by different reactions to produce active amyl alcohol (Dickinson et al., 2000).

In this study, the addition of isoleucine and phenylalanine into longan juice was expected to intensify the production of active amyl alcohol and 2-phenylethyl alcohol, and hence their corresponding acetate esters, in longan wine. Indeed, these alcohols and esters were obtained at significantly higher contents after 21 days of fermentation with the added amino acids than in the control (Table 1). Furthermore, among the quantified volatile compounds (marked by an asterisk in Table 1), isoamyl acetate, 2-phenylethyl acetate, 2-phenylethyl alcohol and ethyl octanoate might be detected organoleptically due to their higher concentrations than the odour threshold values (30, 250, 10000 and $2 \mu \mathrm{g} / \mathrm{L}$ respectively), except for isoamyl alcohol, whose concentration was lower than its threshold of $30000 \mu \mathrm{g} / \mathrm{L}$ (Guth, 2007). Compared to orange wine (Selli et al., 2008),

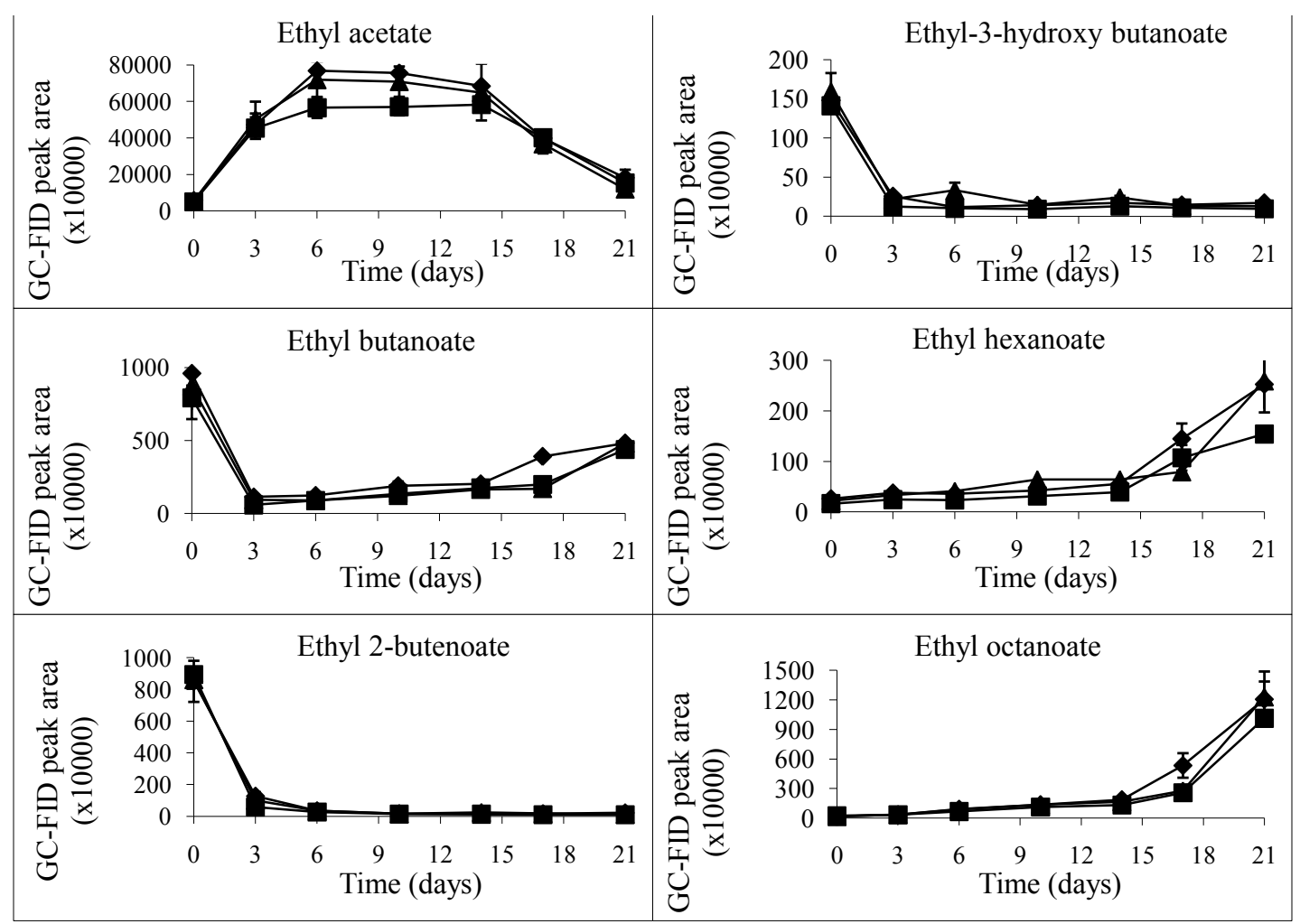

FIGURE 3

Kinetics of ethyl esters during longan juice co-fermentation by S. cerevisiae var. bayanus EC-1118 and W. saturnus var. saturnus CBS254 (ratio of 1:1 000 cfu/mL respectively). Longan juice without added amino acid (control) $(\boldsymbol{\nabla})$, longan juice with added L-isoleucine $(\boldsymbol{\Delta})$, longan juice with added L-phenylalanine $(\boldsymbol{\square})$. 
the amounts of isoamyl acetate in all three longan wines were higher, while the amount of 2-phenylethyl alcohol was strikingly greater in L-phenylalanine-added longan wine; however, active amyl acetate, isoamyl alcohol, ethyl octanoate and octanoic acid were present in lower concentrations. Meanwhile, there was no significant difference between most of the remaining volatile compounds relative to the control, except for isoamyl acetate and hexyl acetate, which constituted significantly lower amounts at a statistical level in the presence of isoleucine and phenylalanine, possibly owing to the diversion of acetyl-CoA to the formation of active amyl and 2-phenylethyl acetates.

Saccharomyces cerevisiae is known to have low alcohol acetyltransferase activity (Yoshioka \& Hashimoto, 1981). Williopsis saturnus var. saturnus was selected for co-culture with $S$. cerevisiae based on the fact that the former yeast has been known to produce high amounts of acetate esters such as isoamyl acetate (Yilmaztekin et al., 2008), which presumably possess high alcohol acetyltransferase activity.

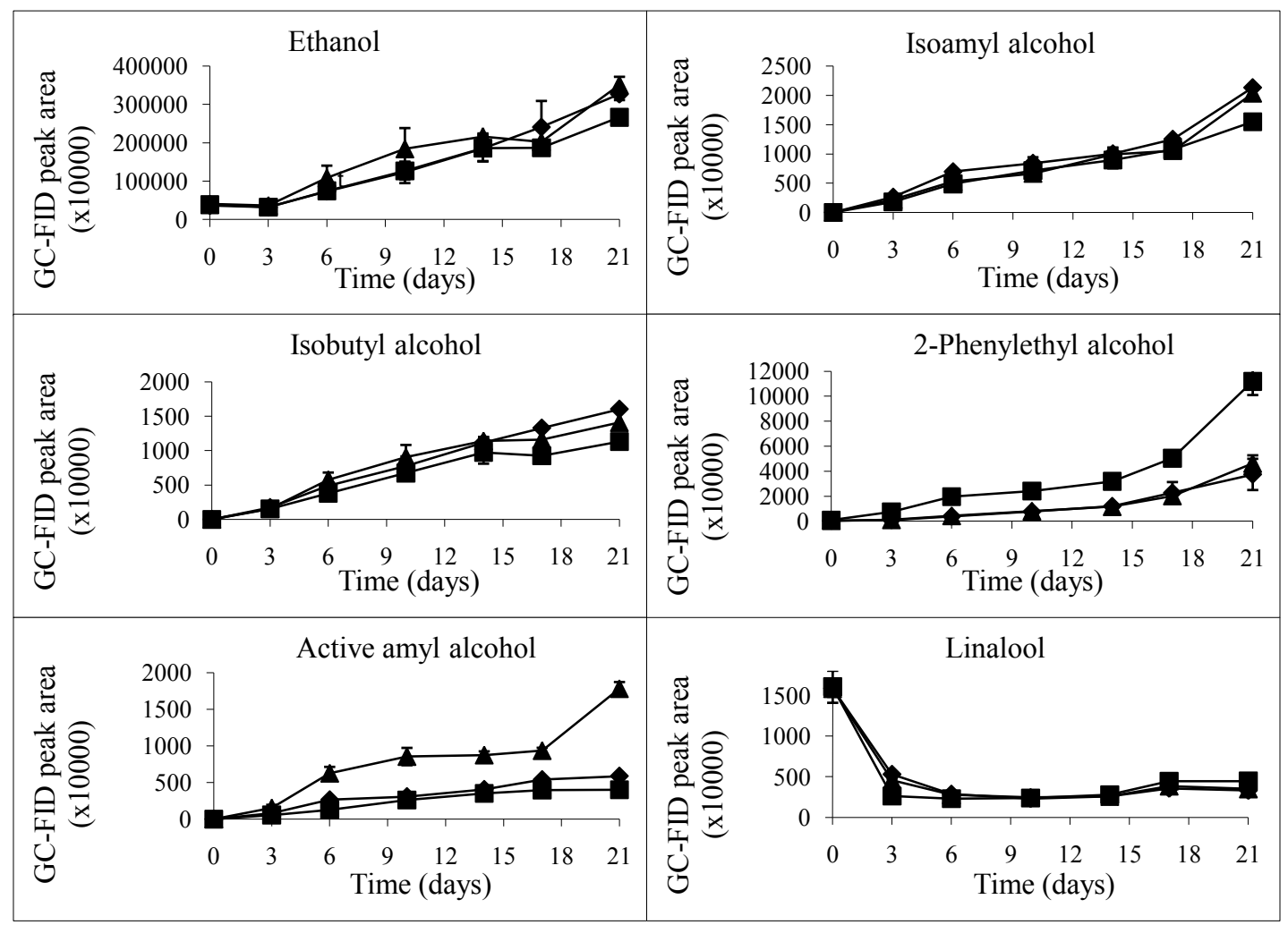

FIGURE 4

Kinetics of alcohols during longan juice co-fermentation by S. cerevisiae var. bayanus EC-1118 and W. saturnus var. saturnus CBS254 (ratio of 1:1 000 cfu/mL respectively). Longan juice without added amino acid (control) $(\boldsymbol{\nabla})$, longan juice with added L-isoleucine $(\boldsymbol{\Delta})$, longan juice with added L-phenylalanine $(\boldsymbol{\square})$.

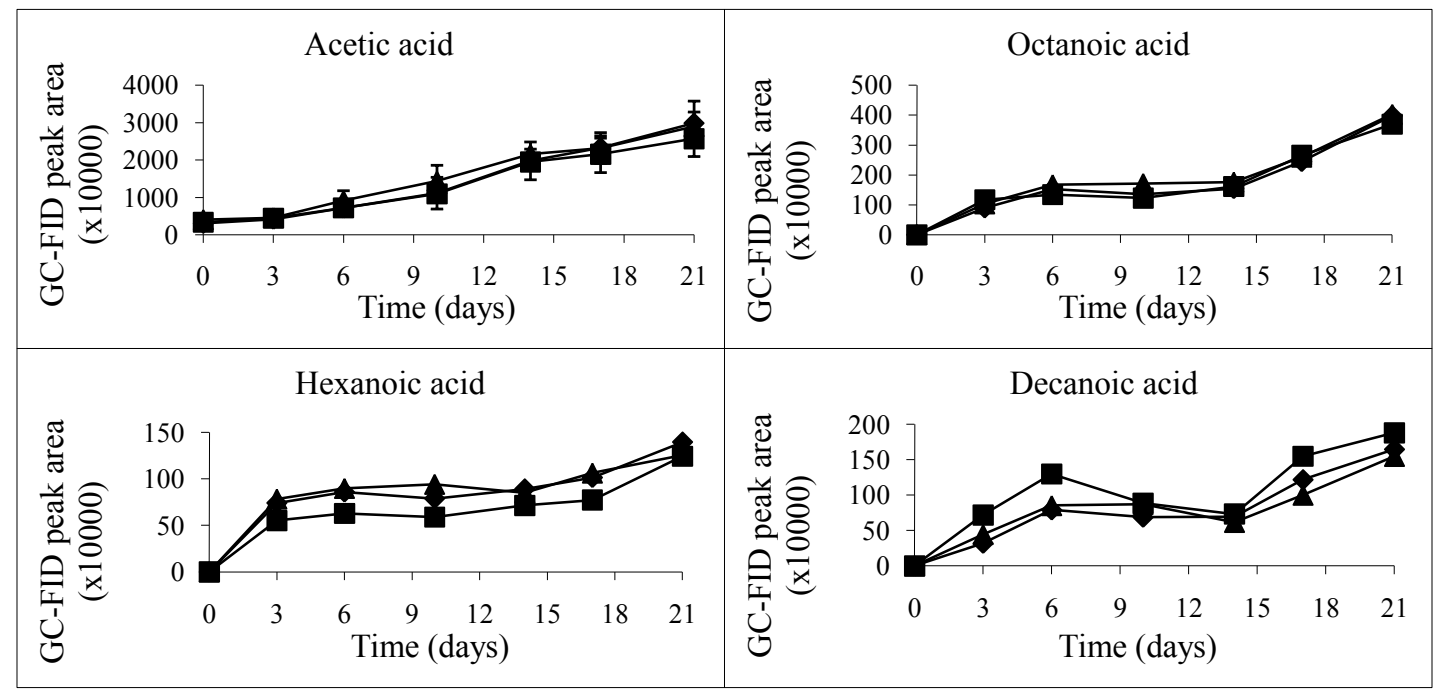

FIGURE 5

Kinetics of organic acids during longan juice co-fermentation by S. cerevisiae var. bayanus EC-1118 and W. saturnus var. saturnus CBS254 (ratio of 1:1 000 cfu/mL respectively). Longan juice without added amino acid (control) $(\boldsymbol{\nabla})$, longan juice with added L-isoleucine $(\boldsymbol{\Delta})$, longan juice with added L-phenylalanine $(\boldsymbol{\square})$. 


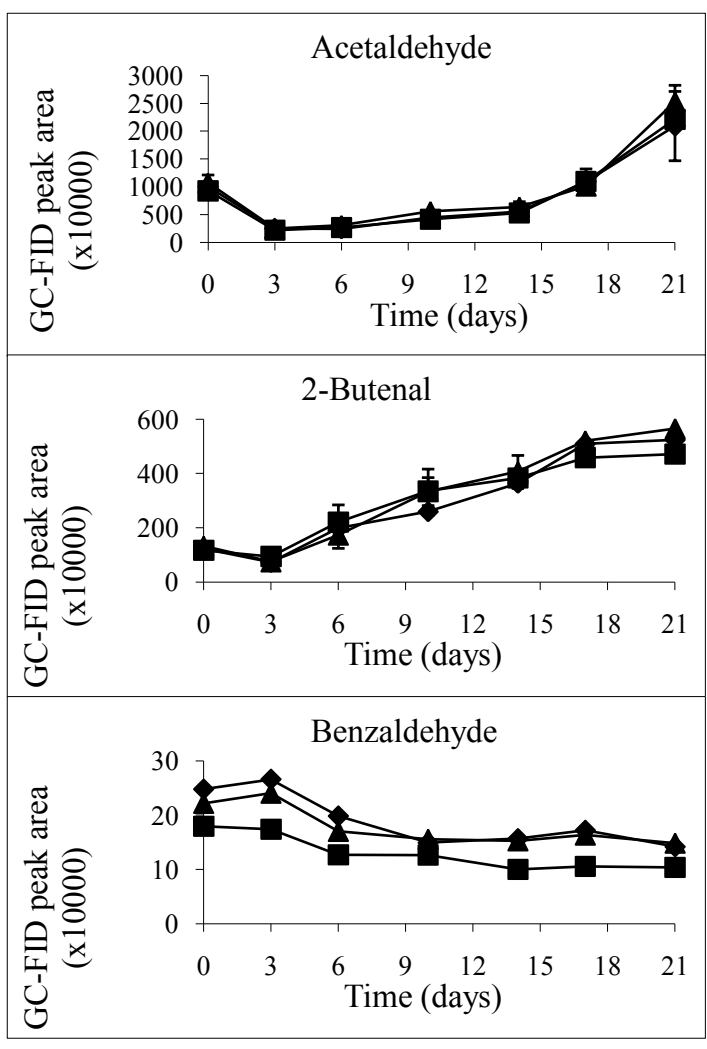

FIGURE 6

Kinetics of aldehydes during longan juice co-fermentation by $S$. cerevisiae var. bavanus $\mathrm{EC}-1118$ and W. saturnus var. saturnus CBS254 (ratio of $1: 1000 \mathrm{cfu} / \mathrm{mL}$ respectively). Longan juice without added amino acid (control) $(\checkmark)$ ), longan juice

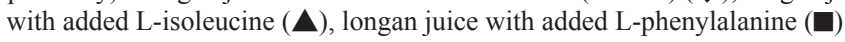

Tropical fruits vary with either the content or the composition of their amino acid profile, since these fruits have generally been known as a low source of protein (Hall et al., 1980). This fact can lead the initial juices to be poor in aroma precursors for fruit winemaking. Hence the addition of exogenous amino acids may be an important approach to direct and control aroma formation. This practice has been applied for making other fruit wines, such as cider, litchi wine in China (Gai et al., 2005; Zhang et al., $2008 \mathrm{~b}$ ), with the aim of supplying the nitrogen source to enhance yeast growth, fermentation ability and ethanol yield.

\section{CONCLUSIONS}

The results of this study show that a mixed culture of S. cerevisiae EC-1118 and W. saturnus CBS254 can be used to enhance the formation of aroma-active compounds, such as active amyl alcohol and its ester active amyl acetate and 2-phenylethanol and its ester 2-phenylethyl acetate (rose-petal aroma), when L-isoleucine and L-phenylalanine respectively are added to longan juice, while either maintaining or decreasing the production of other acetate esters and volatiles. As a result, the addition of a specific amino acid into longan juice can be employed to raise the level of the targeted aroma compounds in longan wine to impart a specific aroma attribute and thus improve the favourability of longan wine among consumers. Further research is required to ascertain the effect of the addition of combinations of selected amino acids in different ratios on targeted aroma compounds and flavour complexity.

\section{LITERATURE CITED}

Chandler, M.A. \& Zydney, A.L., 2005. Clarification of yeast cell suspensions by depth filtration. Biotechnol. Prog. 21, 1552-1557.

Chang, C.Y., Chang, C.H., Yu, T.H., Lin, L.Y. \& Yen, Y.H., 1998. The effect of drying treatment on the flavor and quality of longan fruit. Dev. Food Sci. 40, 353367.

Dickinson, J.R., Harrison, S.J. \& Hewlins, M.J.E., 1998. An investigation of the metabolism of valine to isobutyl alcohol in Saccharomyces cerevisiae. J. Biol. Chem. 273(40), 25751-25756.

Dickinson, J.R., Harrison, S.J., Dickinson, J.A. \& Hewlins, M.J.E., 2000. An investigation of the metabolism of isoleucine to active amyl alcohol in Saccharomyces cerevisiae. J. Biol. Chem. 275(15), 10937-10942.

Dickinson, J.R., Lanterman, M.M., Danner, D.J., Pearson, B.M., Sanz, P., Harrison, S.J. \& Hewlins, M.J.E., 1997. A ${ }^{13} \mathrm{C}$ nuclear magnetic resonance investigation of the metabolism of leucine to isoamyl alcohol in Saccharomyces cerevisiae. J. Biol. Chem. 272(43), 26871-26878.

Dickinson, J.R., Salgado, L.E.J. \& Hewlins, M.J.E., 2003. The catabolism of amino acids to long chain and complex alcohols in Saccharomyces cerevisiae. J. Biol. Chem. 278(10), 8028-8034.

Egli, C.M., Edinger, W.D., Mitrakul, C.M. \& Henick-Kling, T., 1998. Dynamics of indigenous and inoculated yeast populations and their effect on the sensory character of Riesling and Chardonnay wines. J. Appl. Microbiol. 85(5), 779-789.

Etschmann, M.M.W., Bluemke, W., Sell, D. \& Schrader, J., 2002. Biotechnological production of 2-phenylethanol. Appl. Microbiol. Biotechnol. 59(1), 1-8.

Gai, B., Ji, B.P., Zhang, H., Zhou, F. \& Jiang, H., 2005. Study on the utilization of amino acids during the cider making. DOI: CNKI:ISSN:0253-990X.0.200511-009.

Garde-Cerdan, T. \& Ancin-Azpilicueta, C., 2008. Effect of the addition of different quantities of amino acids to nitrogen-deficient must on the formation of esters, alcohols, and acids during wine alcoholic fermentation. LWT 41(3), 501-510.

Guth, H., 1997. Quantitation and sensory studies of character impact odorants of different white wine varieties. J. Agric. Food Chem. 45, 3027-3032.

Hall, N.T., Smoot, J.M., Knight Jr., R.J. \& Nagy, S., 1980. Protein and amino acid compositions of ten tropical fruits by gas-liquid chromatography. J. Agric. Food Chem. 28(6), 1217-1221.

Hansen, E.H., Nissen, P., Sommer, P., Nielsen, J.C. \& Arneborg, N., 2001. The effect of oxygen on the survival of non-Saccharomyces yeasts during mixed culture fermentations of grape juice with Saccharomyces cerevisiae. J. Appl. Microbiol. 91(3), 541-547.

Henick-Kling, T., Edinger, W., Daniel, P. \& Monk., P., 1998. Selective effects of sulfur dioxide and yeast starter culture addition on indigenous yeast populations and sensory characteristics of wine. J. Appl. Microbiol. 84(5), 865-876.

Herrero, M., García, L.A. \& Díaz, M., 2006. Volatile compounds in cider, inoculation time and fermentation temperature effects. J. Inst. Brew. 112(3), 210214.

Hernandez-Orte, P., Ibarz, M.J., Cacho, J. \& Ferreira, V., 2005. Effect of the addition of ammonium and amino acids to musts of Airen variety on aromatic composition and sensory properties of the obtained wine. Food Chem. 89(2), 163174.

Heras-Vazquez, F.J., Mingorance-Cazorla, L., Clemente-Jimenez, J.M. \& Rodriguez-Vico, F., 2003. Identification of yeast species from orange fruit and juice by RFLP and sequence analysis of the 5.8S rRNA gene and the two internal transcribed spacers. FEMS Yeast Res. 3(1), 3-9.

Iwase, T., Morikawa, T., Fukuda, H., Sasaki, K. \& Yoshitake, M., 1995. Production of fruity odor by genus Williopsis. J. Brew. Soc. 90(5), 394-396.

Jiang, Y.M., 1999. Low temperature and controlled atmosphere storage of fruit of longan (Dimocarpus longan Lour.). Trop. Sci. 39, 98-101.

Jolly, N.P., Augustyn, O.P.H. \& Pretorius, I.S, 2003. The effect of nonSaccharomyces yeasts on fermentation and wine quality. S. Afr. J. Enol. Vitic. 24(2), 55-62.

Kim, D.H., Hong, Y.A. \& Park, H.D., 2008. Co-fermentation of grape must by Issatchenkia orientalis and Saccharomyces cerevisiae reduces the malic acid content in wine. Biotechnol. Lett. 30(9), 1633-1638.

Lambrechts, M.G. \& Pretorius, I.S., 2000. Yeasts and its importance to wine aroma - a review. S. Afr. J. Enol. Vitic. 21(1), 97-129. 
Maro, E.D., Ercolini, D. \& Coppola, S., 2007. Yeast dynamics during spontaneous wine fermentation of the Catalanesca grape. Int. J. Food Microbiol. 117(2), 201210 .

Miller, A.C., Wolff, S.R., Bisson, L.F. \& Ebeler, S.E., 2007. Yeast strain and nitrogen supplementation dynamics of volatile ester production in Chardonnay juice fermentations. Am. J. Enol. Vitic. 58(4), 470-483.

Molina, A.M., Swiegers, J.H., Varela, C., Pretorius, I.S. \& Agosin, E., 2007. Influence of wine fermentation temperature on the synthesis of yeast-derived volatile aroma compounds. Appl. Microbiol. Biotechnol. 77(3), 675-687.

Nissen, P., Nielsen, D. \& Arneborg, N., 2003. Viable Saccharomyces cerevisiae cells at high concentrations cause early growth arrest of non-Saccharomyces yeasts in mixed cultures by a cell-cell contact-mediated mechanism. Yeast 20(4), 331-341.

Panjai, L., Ongthip, K. \& Chomsri, N., 2009. Complex fruit wine produced from dual culture fermentation of pineapple juice with Torulaspora delbrueckii and Saccharomyces cerevisiae. Asian J. Food Ag-Ind. 2(2), 135-139.

Pérez-Nevado, F., Albergaria, H., Hogg, T. \& Girio, F., 2006. Cellular death of two non-Saccharomyces wine-related yeasts during mixed fermentations with Saccharomyces cerevisiae. Int. J. Food Microbiol. 108(3), 336-345.

Pina, C., Santos, C., Couto, J.A. \& Hogg, T., 2004. Ethanol tolerance of five non-Saccharomyces wine yeasts in comparison with a strain of Saccharomyces cerevisiae - influence of different culture conditions. Food Microbiol. 21(4), 439447.

Rangkadilok, N., Worasuttayangkurn, L., Bennett, R.N. \& Satayavivad, J., 2005. Identification and quantification of polyphenolic compounds in longan (Euphoria longana Lam.) fruit. J. Agric. Food Chem. 53(5), 1387-1392.

Rapp, A. \& Mandery, H., 1986. Wine aroma. Cell Mol. Life Sci. 42(8), 873-884.
Ribéreau-Gayon, P., Glories, Y., Manjean, A. \& Dubourdieu, D., 2000. Handbook of Enology. The Chemistry of Wine Stabilization and Treatments, vol. 2. John Wiley \& Sons, Chichester

Rojas, V., Gil, J.V., Pinaga, F. \& Manzanares, P., 2003. Acetate ester formation in wine by mixed cultures in laboratory fermentations. Int. J. Food Microbiol. 86(1-2), 181-188

Selli, S., Canbas, A., Varlet, V., Kelebek, H., Prost, C. \& Serot, T., 2008. Characterization of the most odor-active volatiles of orange wine made from a Turkish cv. Kozan (Citrus sinensis L. Osbeck). J. Agric. Food Chem. 56, 227234.

Viana, F., Gil, J.V., Valles, S. \& Manzanares, P., 2009. Increasing the levels of 2-phenylethyl acetate in wine through the use of a mixed culture of Hanseniaspora osmophila and Saccharomyces cerevisiae. Int. J. Food Microbiol. 135(1), 68-74.

Wall, M.M., 2006. Ascorbic acid and mineral composition of longan (Dimocarpus longan), lychee (Litchi chinensis) and rambutan (Nephelium lappaceum) cultivars grown in Hawaii. J. Food Compos. Anal. 19, 655-663.

Yoshioka, K. \& Hashimoto, N., 1981. Ester formation by alcohol acetyltransferase from brewer's yeast. Agric. Biol. Chem. 45(10), 2183-2190.

Yilmaztekin, M., Erten, H. \& Cabaroglu, T., 2008. Production of isoamyl acetate from sugar beet molasses by Williopsis saturnus var. saturnus. J. Inst. Brew. 114(1), 34-38

Zhang, Z.M., Zeng, D.D. \& Li, G.K., 2008a. Study of the volatile profile characteristics of longan during storage by a combination sampling method coupled with GC/MS. J. Sci. Food Agric. 88(6), 1035-1042.

Zhang, B., Zeng, X.A., Chen, Y. \& Yu, S.J., 2008b. Effects of the addition of compound amino acids on the fermentation progress of litchi fruit wine. Liquormaking Sci. \& Technol. 3, 23-25, 28. 